\title{
Dynamic Response Testing in an Electrically Heated Reactor Test Facility
}

\author{
Shannon M. Bragg-Sitton ${ }^{1}$ and T.J. Morton ${ }^{2}$ \\ ${ }^{1}$ NASA Marshall Space Flight Center, Nuclear \& Advanced Propulsion Branch, ER-11, MSFC, AL 35812 \\ ${ }^{2}$ Department of Chemical and Nuclear Engineering, University of New Mexico, Albuquerque, NM 87131 \\ 256-544-6272; Shannon.M.Bragg-Sitton@nasa.gov
}

\begin{abstract}
Non-nuclear testing can be a valuable tool in development of a space nuclear power or propulsion system. In a non-nuclear test bed, electric heaters are used to simulate the heat from nuclear fuel. Standard testing allows one to fully assess thermal, heat transfer, and stress related attributes of a given system, but fails to demonstrate the dynamic response that would be present in an integrated, fueled reactor system. The integration of thermal hydraulic hardware tests with simulated neutronic response provides a bridge between electrically heated testing and full nuclear testing. By implementing a neutronic response model to simulate the dynamic response that would be expected in a fueled reactor system, one can better understand system integration issues, characterize integrated system response times and response characteristics, and assess potential design improvements at a relatively small fiscal investment.

Initial system dynamic response testing was demonstrated on the integrated SAFE-100a heat pipe cooled, electrically heated reactor and heat exchanger hardware, utilizing a one-group solution to the point kinetics equations to simulate the expected neutronic response of the system (Bragg-Sitton, 2005). Reactivity feedback calculations were then based on a bulk reactivity coefficient and measured average core temperature. Similar dynamic test techniques will be applied to a direct drive gas cooled reactor system (DDG), demonstrating the applicability of the testing methodology to any reactor type and demonstrating the variation in system response characteristics in different reactor concepts. Although both system designs utilize a fast spectrum reactor, the method of cooling the reactor differs significantly, leading to a variable system response that can be demonstrated and assessed in a non-nuclear test facility. DDG testing will utilize a higher fidelity point kinetics model that incorporates the complete six delayed neutron groups to control core power transients. Additionally, reactivity feedback will be based on localized reactivity feedback coefficients and several independent temperature measurements taken within the core block. The neutron generation time and individual temperature feedback coefficients will be provided as model inputs. This paper will discuss the methodology that will be implemented in DDG testing and will assess the additional instrumentation needs to implement high fidelity dynamic testing. Dynamic testing is expected to commence early in FY06.
\end{abstract}

\section{INTRODUCTION}

Non-nuclear testing can be used to evaluate the operation of an integrated nuclear system within a reasonable cost and schedule to provide valuable input to the overall system design. Various operational regimes can be studied in non-nuclear testing to validate thermal and thermal hydraulic codes, to assess thermal hydraulic behavior, to characterize stress/strain in the system during operation, and to verify system integration processes. However, because the electrically heated core lacks neutrons, the dynamic neutronic response of the system cannot be fully simulated without the incorporation of additional system models.

In early 2005, dynamic testing was performed on the SAFE-100a test article at NASA Marshall Space Flight Center (MSFC). The SAFE-100a is a resistively heated, stainless-steel heat-pipe (HP)-reactor core segment, coupled to a prototypic gas-flow heat exchanger (HX). Core power transients that were simulated in the electrically heated system tests were controlled by a point kinetics model with reactivity feedback based on a bulk temperature reactivity feedback coefficient and core average temperature. This type of non-nuclear test is expected to provide reasonable approximation of reactor transient behavior because reactivity feedback is very simple in a compact fast spectrum reactor (simple, negative, and relatively monotonic temperature feedback, caused mostly by thermal expansion) and calculations show there are no significant reactivity effects associated with fluid in the HP (the worth 
of the entire inventory of $\mathrm{Na}$ in the core is $<<\$ 1$, so fluid movement and temperature changes will cause very minor effects). In previous SAFE-100 tests, the point kinetics model was based on core thermal expansion via deflection measurements (Bragg-Sitton and Forsbacka, 2004). These tests demonstrated that core deflection was a strong function of how the SAFE-100 modules were fabricated and assembled (in terms of straightness, gaps, and other tolerances). Both the SAFE-100 and 100a were constructed from individual modules that nested together to form a core rather than being constructed from a monolithic block. To remove the added variable of how the SAFE-100a expands with varying temperature as compared to a different concept, it was decided to use a temperature based feedback model (based on several thermocouples placed throughout the core). The bulk core temperature feedback coefficient for most reactors of this class is on the order of -0.1 to -0.2 cents per degree $\mathrm{K}$.

The test matrix applied in SAFE-100a testing included changes in coolant flow rate to simulate system load variations and step reactivity insertions and decreases. The system responded as "expected" for all induced transients: increased / decreased coolant flow rate with reactivity feedback, increased / decreased coolant flow rate without reactivity feedback (done for comparison), and positive / negative reactivity insertion. "Expected" implies that the transient response was typical of any simple reactor, whether it be cooled by a pumped fluid (e.g. gas or liquid metal) or by HPs. Figure 1 shows the system response to a series of transients in which the system load was increased several times (represented by mass flow increase through the $\mathrm{HX}$ ), followed by a step reactivity insertion of \$0.06. In-depth discussion of the SAFE-100a system response can be found in Bragg-Sitton (2005).

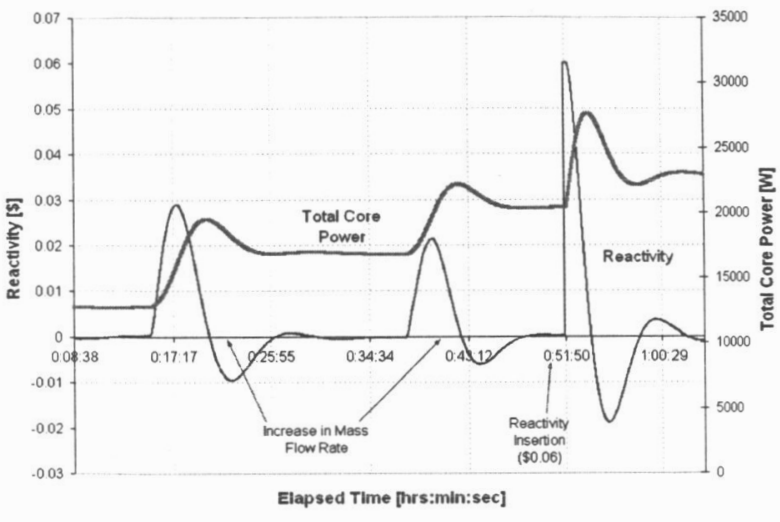

(a)

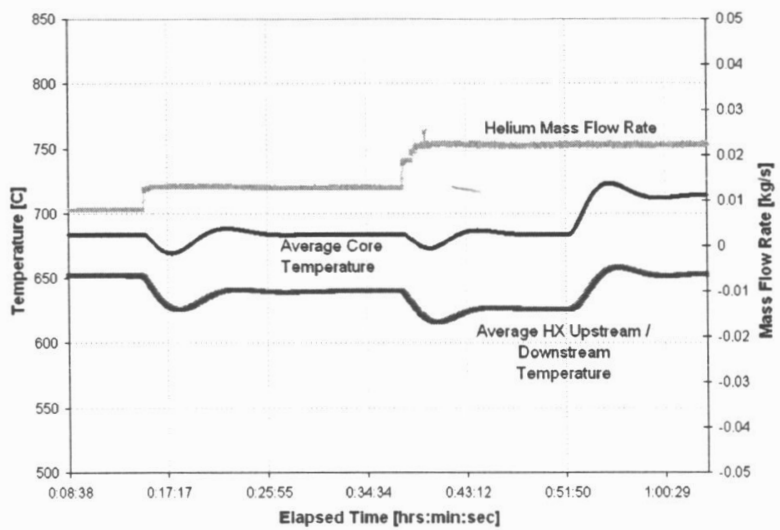

(b)

FIGURE 1. Dynamic System Testing of the SAFE-100a with Simulated Reactivity Feedback: (a) Core Thermal Power and Calculated Reactivity and (b) HX Mass Flow Rate, Average Core Temperature, and Average Heat Pipe Temperatures Upstream and Downstream of the HX.

During the applied transients, the HPs appear to act as a very good conductor with relatively little thermal inertia, such that system response would be expected to be very similar in a reactor design in which gas directly removes power from the core, as in a direct drive gas cooled reactor which does not incorporate an intermediate heat exchanger. The time scale of the transient is dictated mostly by the thermal inertia of the core, and to a smaller extent by the HPs and HX for the SAFE-100a. In each transient applied to the SAFE-100a, the peaks and/or valleys of the power and temperature occurred within 3 to 5 minutes after transient initiation, followed by modest oscillations until the system stabilized at a new steady state condition approximately 20-30 minutes after the transient was initiated. The magnitude of power and temperature overshoots were also modest (e.g. $\sim 15 \mathrm{~K}$ temperature overshoot for a $\$ 0.10$ reactivity insertion), although the magnitude of overshoot is not of great meaning because the SAFE-100a is a low power density core with non-prototypic thermal inertia.

Similar dynamic test methodology will be applied to a direct drive gas cooled reactor (DDG) design that is currently under test at NASA MSFC. Designed by engineers at Sandia National Laboratory and the Los Alamos National Laboratory, the DDG was previously tested to assess basic flow characteristics and system pressure drop (Godfroy, Kapernick and Bragg-Sitton, 2004). This core is currently being refurbished to incorporate additional instrumentation to measure core block temperatures and to replace the existing graphite heater elements that have 
undergone a significant amount of thermal cycling during previous check-out tests. Instrumentation will be positioned to provide extensive characterization of the core block temperature, both axially and radially, and to monitor coolant gas temperature in regions of interest. Details of the proposed instrumentation will be discussed. More detailed characterization of core temperatures will allow implementation of a higher fidelity neutronics model that incorporates multiple reactivity feedback coefficients as a function of various core temperature measurements. Additionally, investigation of deflection measurement techniques for application inside a pressure vessel has commenced. Potential applicability of these methods will be discussed.

\section{SYSTEM MODEL: POINT REACTOR KINETICS}

Reactor dynamics can be modeled using the point kinetics equations (PKE), which can be derived from transport and diffusion theory (Hetrick, 1971). The PKE representation provides only an approximate model of the reactor; it does not provide a mechanism to describe neutron energy effects or structural details in a heterogeneous reactor. Because fast reactors are relatively small in size and do not contain any moderator to slow the neutrons to a lower energy, the PKE are a good approximation for the dynamics in a fast reactor. In the absence of an external source and written in terms of reactor thermal power, the PKE are given by:

$$
\begin{gathered}
\frac{d P}{d t}=\frac{(\rho-\beta)}{\Lambda} P+\lambda_{i} C_{i}, \\
\frac{d C_{i}}{d t}=\frac{\beta_{i}}{\Lambda} P-\lambda_{i} C_{i},
\end{gathered}
$$

where the reactivity, $\rho$, is given by:

$$
\rho=\frac{k-1}{k},
$$

and $k$ is the effective neutron multiplication factor. Reactivity is often expressed in units of dollars. The reactivity scale between zero and $\beta$, the total delayed neutron fraction, is divided into 100 cents, where 100 cents of reactivity is equal to one dollar. Hence, the reactivity expressed in units of dollars is given by $\rho / \beta$.

Studies of reactor dynamics generally recognize six distinct groups of delayed neutrons. Approximate solutions to the PKE can be found using a smaller number of groups to represent the delayed neutron population. The dynamic model applied in the previous application of simulated reactivity feedback on the SAFE-100a included a simple dynamic model having one group of delayed neutrons, applying a weighted average decay constant and the total delayed neutron fraction. The one group decay constant $(\lambda)$ is calculated as a weighted average of the six individual decay constants. A one group representation simplified the computational model, speeding the real-time implementation of simulated reactivity feedback during test. However, to improve the fidelity of the neutronic model, a more detailed PKE representation should be employed.

A complete six-group model, requiring seven ordinary differential equations, will be applied in DDG testing. The individual decay constants and delayed neutron fractions for a fast spectrum reactor, as reported by Hetrick (1971), are provided in Table 1. To maintain real-time implementation of the neutronics model, high speed computing must be employed. Potential options for implementing real-time system control will be discussed in a later section.

A simple model of a point reactor with feedback was applied in approximating the temperature reactivity feedback in the SAFE-100a. In that application, a single bulk reactivity feedback coefficient, $\alpha_{T}$, was applied to the entire core. The feedback calculation was then made based on bulk core temperature changes, determined by calculation of the average core temperature at the axial core centerline. In this simple case, the total reactivity of the reactor core is given by Eq. (2):

$$
\rho=\rho_{o}+\alpha_{T}(\Delta T)
$$


TABLE 1. Delayed Neutron Properties for Fast Fission of ${ }^{235} U$ (Hetrick, 1971).

\begin{tabular}{|c|c|c|}
\hline $\begin{array}{c}\text { Delayed Neutron } \\
\text { Group }\end{array}$ & $\begin{array}{c}\text { Decay Constant } \\
\boldsymbol{\lambda}_{\boldsymbol{i}}[\mathbf{s e c}]^{-1}\end{array}$ & $\begin{array}{c}\text { Relative Yield } \\
\boldsymbol{\beta}_{\boldsymbol{i}} / \boldsymbol{\beta}\end{array}$ \\
\hline 1 & 0.0127 & 0.038 \\
\hline 2 & 0.0317 & 0.213 \\
\hline 3 & 0.115 & 0.188 \\
\hline 4 & 0.311 & 0.407 \\
\hline 5 & 1.40 & 0.128 \\
\hline 6 & 3.87 & 0.026 \\
\hline Total Decay Constant, $\lambda=0.0767 \mathrm{sec}^{-1}$ & \\
\hline Total Delayed Neutron Fraction, $\beta=0.00642$ \\
\hline
\end{tabular}

where $\alpha_{T}$ is the temperature coefficient of reactivity,

$$
\alpha_{T}=\frac{d \rho}{d T},
$$

and $\rho_{o}$ is the initial steady-state reactivity before a transient is applied in a reactor power. In the previous application, $\alpha_{T}$ incorporated all reactivity feedback effects due to fuel and structural temperature changes (including Doppler broadened cross sections and thermal expansion effects). However, if highly precise temperature (and possibly deflection) measurements can be made, each of these individual effects on reactivity can be assessed separately in the feedback model. For example, Eq. (2) would be expanded to include several components of reactivity feedback:

$$
\rho=\rho_{o}+\rho_{d L}(d L(T))+\alpha_{T, \text { fuel }}\left(\Delta T_{\text {fuel }}\right)+\alpha_{T, \text { block }}\left(\Delta T_{\text {block }}\right)+\alpha_{T, \text { reflector }}\left(\Delta T_{\text {reflector }}\right)+\cdots
$$

In Eq. (4), temperature feedback was broken into components based on current fuel, core block, and reflector temperatures. In non-nuclear testing, fuel temperature changes must be approximated from measured temperatures in the electric heating element or heater sheath, which is equivalent to the fuel clad structure. Additionally, the nonnuclear test article may not incorporate a full reflector. In this case, the reflector temperature can be inferred from measured temperatures near the periphery of the core block and at the periphery of the pressure vessel in the case of a reactor cooled by a pumped fluid (as in the DDG).

One might choose to base reactivity feedback calculations solely on temperature measurements in various locations, or reactivity feedback can be determined from measured deflection in various regions of the core. Monte Carlo calculations can be performed to assess the reactivity worth of various core deflections that occur due to elevated temperature during core operation. The largest reactivity worth is expected to be due to fuel expansion with temperature, which can be further separated into radial and axial expansion. However, in the absence of an electric heater that closely mimics the dynamic characteristics of nuclear fuel (including thermal expansion), deflection based reactivity feedback will likely need to be based on measured deflection of the core block, as was performed in SAFE-100 testing (Bragg-Sitton and Forsbacka, 2004). Options for measuring the deflection inside the DDG pressure vessel will be discussed in a later section.

\section{TEST ARTICLE DESCRIPTION}

The direct-drive gas-cooled reactor (DDG) is a gas-cooled, UN-fueled, pin-type fast reactor designed to use HeXe gas flowing directly into a recuperated Brayton system to produce electricity for nuclear electric propulsion (Wright and Lipinski, 2003). The DDG, which consists of 37 fuel pins in a block matrix, is designed to operate at $32 \mathrm{kWt}$. This is a reduced power version of a full scale design, similar to the SAFE-30 test series that preceded the SAFE100 and 100a test series (VanDyke et al., 2001). Each fuel pin is surrounded by an annular flow channel, which provides direct heat removal by flowing gas. The DDG design was initially provided by Sandia National 
Laboratories but was transferred to Los Alamos National Laboratories for additional testing support. The DDG is constructed entirely of stainless steel. The core block is a $53.3 \mathrm{~cm}(21$ ") long, $16.5 \mathrm{~cm}(6 \mathrm{1} / 2$ ") (flat-to-flat) hexagonal, solid stainless steel block with a pattern of $37,3 / 4$ " diameter holes gun-drilled through the length. A close-up of the core inlet region, with graphite electric heaters (thermal simulators) installed, is shown in Figure 2.

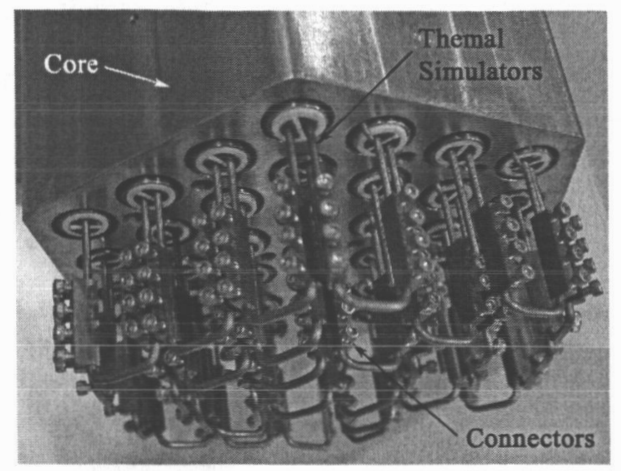

FIGURE 2. Core Block Inlet End with Graphite Heater Elements Installed.

The DDG was designed to operate at $2.4 \mathrm{MPa}$ ( 350 psia) HeAr gas coolant with core inlet temperature of $650 \mathrm{~K}$, core exit temperature of $850 \mathrm{~K}$, and bulk coolant flow of $0.1 \mathrm{~kg} / \mathrm{s}$. Due to the large cost of Xe gas, HeAr, which can duplicate the $\mathrm{HeXe}$ gas requirements, can be used in early tests to reduce the cost of the test program. Initial testing of the DDG was performed with nitrogen gas to allow early system checkouts and verification of the test matrix for early problem identification, with gas flow parameters selected based on a Reynolds number comparison. The test article is also plumbed to allow testing with any desired mixture of He and Ar. In the DDG design, the coolant gas enters the upper bonnet and is directed via a manifold to six 1" diameter inlet tubes located on the upper bonnet assembly, as shown in Figures 3 and 4. The gas then flows down between the pressure vessel wall (called the downcomer region), keeping the vessel wall at a constant lower temperature and providing gas insulation of the core; the insulation layer is constructed by including a metal "shield" between the core block and the pressure vessel wall, causing a stagnant gas layer to form between the core block and the metal shield. At the base of the pressure vessel, the gas collects in a plenum and is directed back up through 37 annular flow channels in the core. Flow parameters are selected such that the gas is heated approximately $200^{\circ} \mathrm{C}$ through the core. Exit gas is then collected in an exit plenum where the flow is recombined and allowed to exit through the upper bonnet center exit pipe.

\section{INSTRUMENTATION PLAN}

Initial check-out testing of the DDG was performed with minimal instrumentation of the DDG test article. The primary intent of these initial tests was to verify core operation, to assess gas flow temperatures, and to experimentally determine the core pressure drop in order to validate thermal hydraulics codes. A majority of the thermocouples (TCs) were installed to measure gas flow temperature in various regions. A few TCs were tack welded to the core block surface to obtain an approximate block temperature, but the temperature measurements from these TCs likely provided a combined block and flow temperature. Rather than being embedded in the core block, these TCs were mounted to the upper block surface where gas flow provided some cooling of the TC junction. No temperature measurements were made in the internal block region. During refurbishment of the DDG core, necessary to install new electric heaters for the next phase of testing, significant modifications will be made to the core instrumentation to provide the necessary data for implementation of simulated reactivity feedback in dynamic system testing.

\section{Temperature Measurement}

Various options for temperature measurement in the DDG core block and heater elements are currently being investigated. Ideally, temperature measurements would be made in the gas flow regions, within the core block, and between the electric heater and the sheath/fuel clad to obtain an estimate of fuel temperature. Gas flow temperatures were measured in the initial DDG testing and will again be measured in planned tests. Eight type-K thermocouples 
will be positioned in key regions of interest to assess gas temperature: two in the gas blanket insulation region, two in the flowing gas downcomer region, two in the gas inlet plenum, and two in the gas outlet plenum. Additional thermocouples will be placed throughout the core block to fully characterize the block temperature both radially and axially. Methods of measuring the temperature near the heater element are also being investigated.

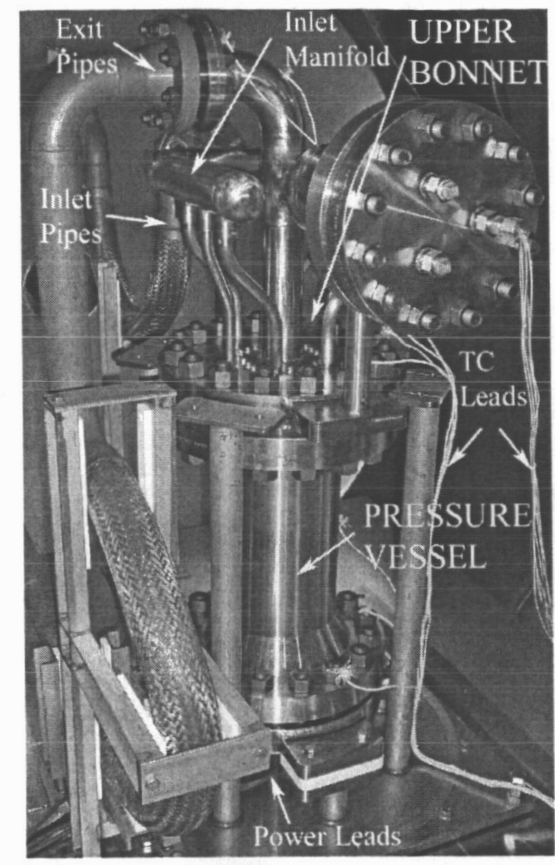

FIGURE 3. DDG Test Article Installed in Vacuum Chamber.

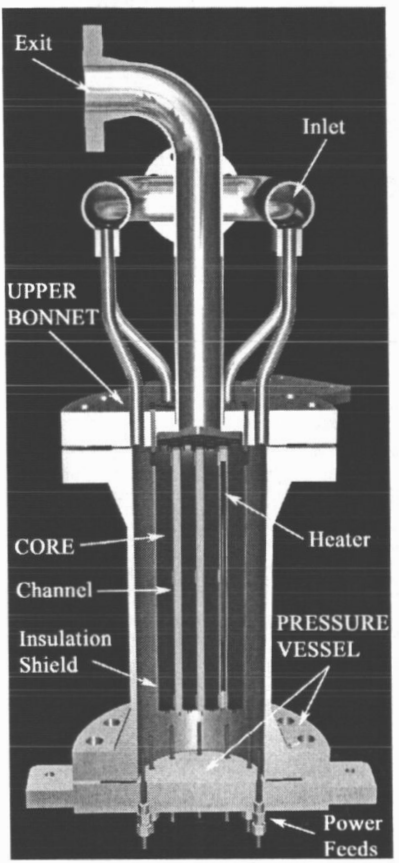

FIGURE 4. Graphical Rendering of the DDG.

\section{Core Block Temperature Measurements}

A series of sheathed, type $\mathrm{K}$ thermocouples will be installed throughout the DDG core block to fully characterize axial and radial temperature distribution. To minimize potential damage to the core block, machining of the block necessary for TC placement will be kept to a minimum. To characterize the axial temperature distribution, it is desirable to obtain temperature measurements along the core centerline and periphery at several axial locations. To accomplish this, the central heating element will be removed (reducing the number of heaters and flow channels to 36 , representing a configuration in which a central safety rod might be used) and will be replaced with an inconel sheathed axial profile probe. Manufactured by Omega, these probes can be purchased in whatever length and diameter are desired, with the number and location of thermocouples located along that length also specified by the customer. The DDG core block is $52.7 \mathrm{~cm}(20.75 ")$ in length and is equipped with $50.8 \mathrm{~cm}\left(20.0^{\prime \prime)}\right.$ heater elements. The bore holes in the core, which accommodate a heater, sheath, and annular flow path, are $1.9 \mathrm{~cm}\left(0.75^{\prime \prime}\right)$ in diameter. For DDG testing, a $51.4 \mathrm{~cm}(20.25 "), 1.7 \mathrm{~cm}(0.67 ")$ diameter probe will be employed, with five TCs located at the following axial positions: $2.54 \mathrm{~cm}, 14.5 \mathrm{~cm}, 26.4 \mathrm{~cm}, 38.3 \mathrm{~cm}, 50.2 \mathrm{~cm}\left(1.0^{\prime \prime}, 5.69 ", 10.375^{\prime \prime}, 15.06 "\right.$, $19.75^{\prime \prime)}$. A $1.3 \mathrm{~cm}(1 / 2 ")$ metal plug installed in the central channel at the gas exit end of the core will prevent gas flow along the axial profile probe. Positioning the first and last TCs one inch from either end of the core block ensures that they will be located in the heated region, accounting for the slightly shorter heating element $(50.8 \mathrm{~cm}$ vs. $52.7 \mathrm{~cm}$ core block). The axial centerline probe will be complemented by five TCs located on the core periphery, embedded approximately $0.64 \mathrm{~cm}(1 / 4 ")$ into the core block. The axial positions of these TCs will be selected to correspond with the TC positions in the profile probe, as shown in Figure 5.

The radial temperature profile will be obtained by embedding TCs into the core block at both the inlet and outlet surfaces. The thermocouple positions are selected to provide radial profiles of interest in two dimensions at each core surface. These TCs will be embedded approximately one inch into the block to ensure that they are located in the heated region, as discussed above. Complete radial profiles at either end of the core block and complete 
measurement of the axial profiles along the centerline and the periphery will allow the radial temperature profile to be interpolated at any axial position. Proposed TC locations are superimposed on photos of the core block in Figure 6. The proposed positions on the gas exit end of the core take advantage of existing holes in the core block that are not currently being used; these will be bored to the desired one inch depth for TC placement. Dashed lines in Fig. 6 show the approximate temperature profile obtained from "surface" TC measurements.

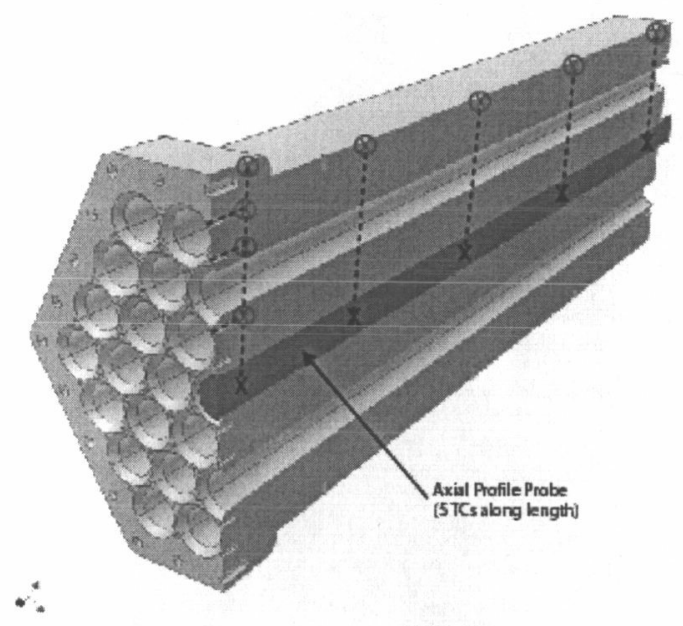

(a)

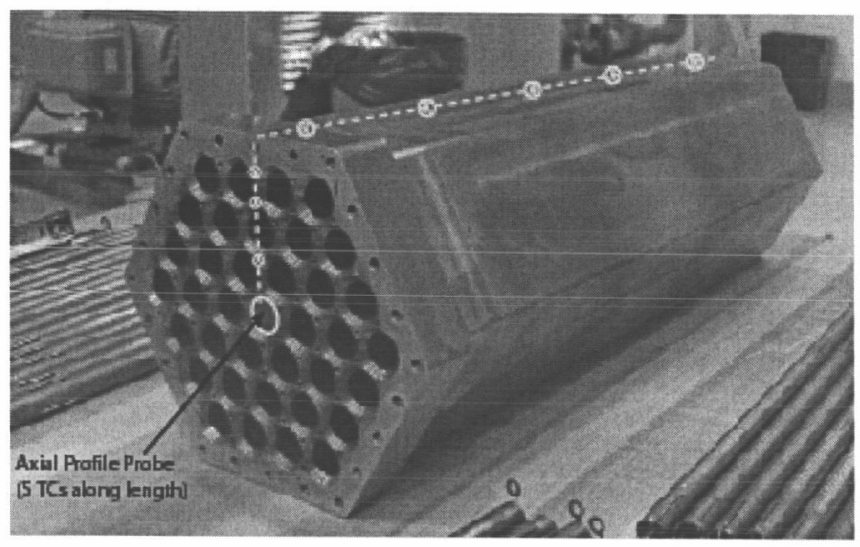

(b)

FIGURE 5. (a) Graphical Rendering and (b) Hardware Representation of Axial Thermocouple Placement in the DDG Core Block with the Central Element Replaced by an Axial Profile Probe; TC Locations are Denoted by Xs.

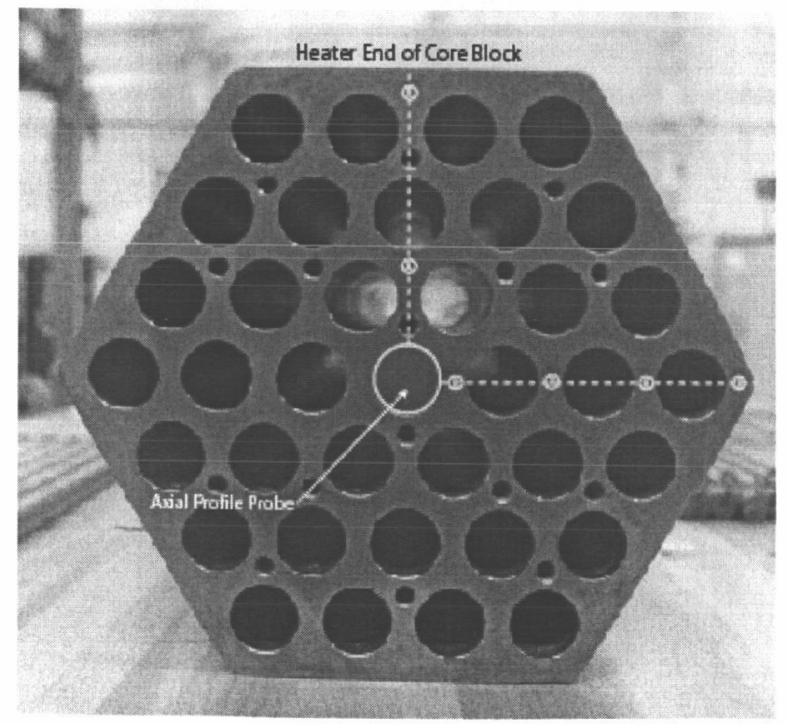

(a)

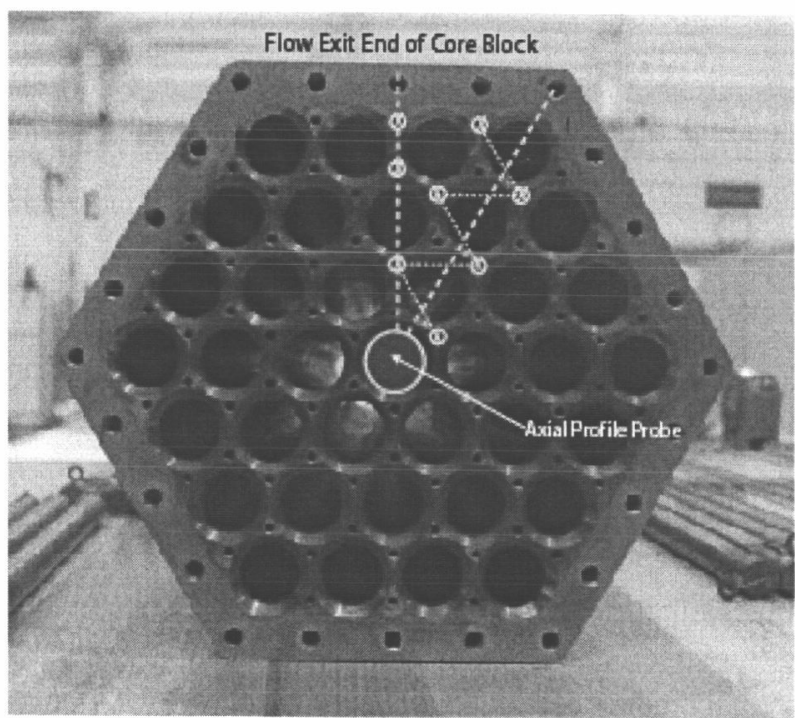

(b)

FIGURE 6. Radial Thermocouple Placement on the (a) Flow Entrance (Heater End) and (b) Flow Exit Ends of the DDG Core Block; the Central Element is Replaced by an Axial Profile Probe and TC Locations are Denoted by Circled Xs.

\section{Fuel Temperature Measurment}

To implement detailed reactivity feedback, it is also desirable to measure temperature in the heater region to approximate the corresponding fuel temperature at a given power level. This requires a temperature measurement 
device to be inserted in the $0.7 \mathrm{~cm}$ gap between the heater element and the sheath structure, shown in Figure 7; this gap is currently filled by an alumina space ring at three axial locations. This may be accomplished with very fine thermocouples embedded in the insulator rings on the heater element, or fiber optic probes might be employed.

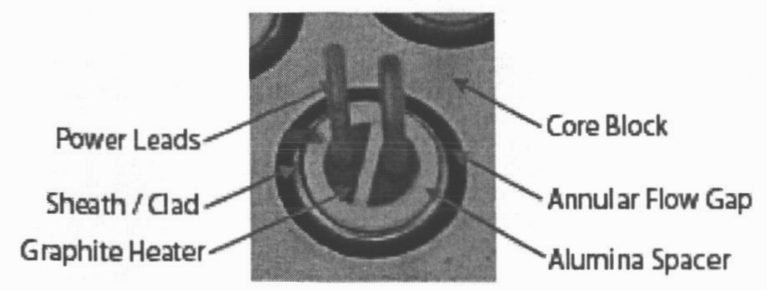

FIGURE 7. Heater Element Placement Inside DDG Core Block.

Fiber optic temperature probes developed by Luna Innovations, Inc. are constructed with Bragg gratings spaced evenly along the sensing length to obtain detailed temperature profiles. These fiber optics have a temperature limitation of $1000{ }^{\circ} \mathrm{C}$ for testing over extended times and may not survive over long test times at elevated temperature in the DDG core. Luna fiber optic probes tested on the SAFE-100 demonstrated superior ability in generating three dimensional temperature profiles of the core (Stinson-Bagby and Fielder, 2004). Luna is currently working to extend the maximum operating temperature of the fiber optic sensors, which may qualify them for use as embedded temperature instrumentation in future advanced heater elements. Previous tests with Luna fiber optics were conducted by Luna, who brought the support hardware necessary to obtain and record data from the fiber optic probes to NASA's Marshall Space Flight Center for temporary use. The EFF-TF is currently working to obtain an in-house capability to use fiber optic sensing techniques in future testing applications.

At present, standard thermocouples are being investigated to measure temperature along the heating element. Because TCs are relatively inexpensive and are available in a wide range of sizes and configurations, it was feasible to test a proposed configuration in a relatively short time frame. Six $0.254 \mathrm{~mm}(0.010 ")$ diameter, inconel-sheathed type $\mathrm{K}$ thermocouples with an ungrounded junction were procured to assess their applicability in DDG testing. Graphite heater elements used in the DDG testing are $0.985 \mathrm{~cm}(0.375 ")$ in diameter and are single ended (designed such that both inlet and outlet power leads are on a single end). These fit inside a $1.4 \mathrm{~cm}(0.55$ ") diameter sheath, the outer diameter of which forms the annular flow path. The heater is separated from the sheath using three alumina spacer rings to prevent contact between the graphite element and the metal sheath. The junction end of a TC was bonded to the graphite element at each alumina ring using alumina cement to obtain three temperature measurements along each element, as shown in Figure 8. During test, several problems were noted with this configuration. Because the TC sheath is made of an electrically conductive material (inconel), bonding the sheath to the heater in more than one location set up a parallel current path in the inconel sheath. The current required to operate the heater element caused the sheath to vaporize and the 52 AWG TC wires did not survive. Future investigation will consider alternate methods of embedding thermocouples within the heater elements to obtain approximate "fuel" temperature measurements.

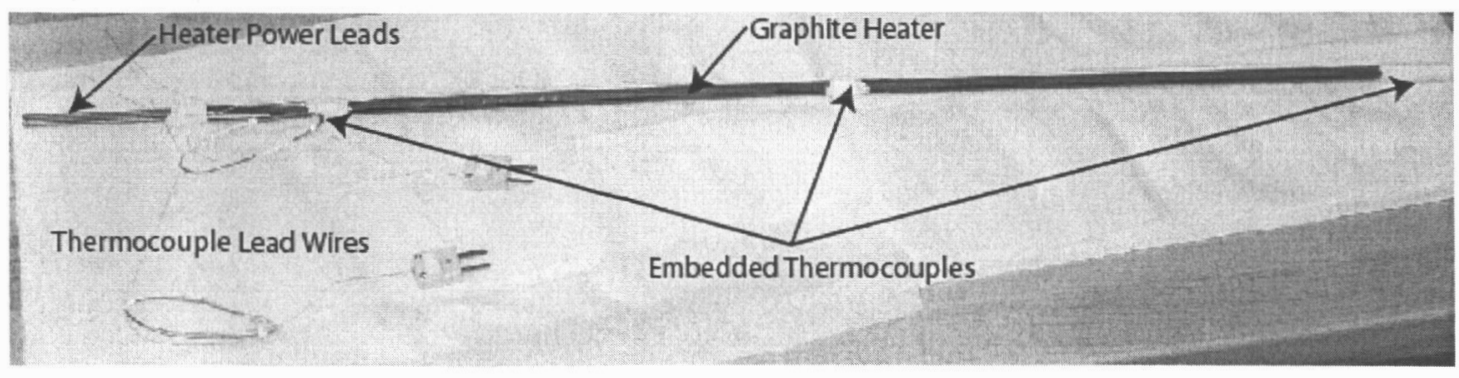

FIGURE 8. Thermocouple Location on Graphite Heater Element for Preliminary Testing. 


\section{Deflection Measurement}

Reactivity feedback based on core deflection measurements was implemented in SAFE-100 hardware testing (Bragg-Sitton and Forsbacka, 2004). Because the SAFE-100 did not require a pressure vessel, visual access to the core was maintained. In this case, deflection could be measured using high resolution photography. Mounted outside the vacuum chamber, imagery from a 6.3 mega-pixel high resolution camera, composed of a charged couple device (CCD) and a camera lens, was used to assess core deflection as a function of temperature by scanning multiple cross sections along the axial dimension of the core. The SAFE-100 was located on an immobile stand inside the chamber, and the CCD imaging system had a clear view of the core through a fused silica viewing port (the device may be installed in the downcomer region, where inlet gas flow is $\sim 450 \mathrm{~K}$ ).

Deflection measurement becomes increasingly complex when visual access is not readily available. Because the DDG must be tested inside a pressure vessel, novel deflection measurement techniques must be investigated. Displacement can be measured using an optical proximity sensor (e.g. a laser range finder), a differential variable reluctance transducer (DVRT), or a non-contact inductance proximity sensor. Each of these was evaluated for its applicability in DDG testing. The next stage of DDG testing will be performed at approximately $0.34 \mathrm{MPa}(50 \mathrm{psi})$ in order to accommodate a Brayton energy conversion system that may be incorporated with the DDG hardware. This reduced pressure condition somewhat relaxes the requirements for a deflection measurement system, but the gas temperature will be approximately the same as in previous testing.

Initial investigation indicates that optical proximity sensors or laser range finders may not be a reliable method of deflection measurement. In this application, the sensor would be directed at the external core block surface. This would require a window to be installed in the DDG pressure vessel, but it is difficult to find ports with viewing windows rated for the experimental pressure and temperature conditions. Additionally, these instruments are dependent on the emissivity of the surface being investigated. As the core surface temperature increases, the emissivity of the stainless steel block changes, such that the accuracy of the sensor over time can be compromised.

Differential variable reluctance transducers (DVRTs) were previously investigated for application to the SAFE-100 and SAFE-100a test articles. A DVRT uses a plunger that is in contact with the core surface. Deflection is then determined by measuring differential reluctance between magnetically shielded DVRT coils. Unfortunately, very few of the commercially available units are rated for high temperature operation $\left(>773 \mathrm{~K}\left(500^{\circ} \mathrm{C}\right)\right.$ ). Although the sensor would not be in direct contact with the hot core block, the plunger conducts heat to the sensor and can cause the operating temperature to be exceeded.

Eliminating direct contact with the hot core block surface reduces the temperature that will be seen by a sensor housing. This can be accomplished using a non-contact inductance proximity sensor. These devises are commercially available for operation at pressure and at elevated temperatures. RDP Electrosense high temperature non-contact transducers employ an inductive proximity position sensor principle and will operate with any ferritic material. The output of the displacement transducer varies as the gap between the front face of the transducer and the target changes. These sensors have electron beam welded joints and are rated for operation from full vacuum up to $20 \mathrm{MPa}(2900 \mathrm{psi})$ and up to $873 \mathrm{~K}\left(600{ }^{\circ} \mathrm{C}\right)$. The RDP sensor can be purchased with a measurement range of up to $6 \mathrm{~mm}$, with a quoted linearity of $\pm 0.05 \%$ of full scale. Sensor model PY256C has been ordered to determine potential applicability in DDG testing. The sensor head itself is $4.8 \mathrm{~cm}$ in length with a maximum diameter of 2.8 $\mathrm{cm}$, making it a tight fit between the core and the pressure vessel. The unit must be calibrated for operation in the desired environment prior to implementation, somewhat complicating its use. Initial calibration and testing is expected to commence in early FY06 after the unit has been received.

\section{CONTROL METHODOLOGY: IMPLEMENTATION}

Specialized LabView programs have previously been used to implement all aspects of system operation. One visual interface provides power supply control, while a second monitors core temperatures and controls the gas supply to remove heat from the core (either for the heat exchanger in the SAFE-100a or the direct gas flow through the DDG). In order to implement reactivity feedback control, the LabView control program additionally implements a solution to the PKE to govern core power response to the transient. 
In SAFE-100a testing, which implemented the PKE with one group of delayed neutrons, the neutronic model included only two differential equations. The simplicity of this model allowed the solution to the system of equations to be pre-calculated and programmed into LabView in algebraic form, minimizing the computation time required by the LabView data acquisition and control system. The solutions to the PKE were then implemented in real time at each iteration of the control loop. All thermocouples installed in the test article were scanned at a rate of $5.8 \mathrm{~Hz}$ (approximately once every $100 \mathrm{~ms}$ ). Communication between the control computer and the power supply controllers can take an additional $150 \mathrm{~ms}$. To account for the total communications delay of up to $250 \mathrm{~ms}$, the control time was set to $500 \mathrm{~ms}$ to prevent the control algorithm from becoming out of synch with the data acquisition or from overloading the power controllers.

DDG testing will require increased computation time to solve a system of seven differential equations at each iteration of the control loop. To accomplish this without impacting the control time, investigation has been initiated to determine ways in which the computational efficacy can be improved. One possible solution is to develop the neutronics model in a Simulink code that can be operated on a separate computer that interfaces back to the governing LabView data acquisition and control code. This arrangement requires that the dynamic model computer run $\mathrm{xPC}$ target, an additional MatLab product. The control computer can then communicate with the model computer, sending updated data to the Simulink model at specified time steps, which then returns the new power solution to the PKE for application to the system. Both UDP communications and shared memory are being investigated as potential communication options between the target and control PCs. Separate model and control computers were selected over a configuration in which Simulink and LabView would be run on the same PC to minimize complexity within the LabView program that would be required to directly interface with Simulink. Additionally, using a single processor places a fundamental limit on the complexity that can be added to the Simulink model while maintaining reasonable calculation rates. The capability of updating data acquisition and control information at $100 \mathrm{~ms}$ time steps is currently being investigated.

\section{CONCLUSIONS}

Integrated system tests with simulated reactivity feedback on the SAFE-100a demonstrated the potential benefits to system operation with simulated neutronic response based on measured system conditions. By simulating dynamic system response, a better understanding can be gained with respect to system integration issues, system response times and response characteristics and overall system design, potentially allowing for design improvements prior to large fiscal and manpower investments into a nuclear test program. Plans are underway to implement similar test methodology on direct gas cooled reactor under test at the EFF-TF. This implementation will incorporate higher fidelity neutronic models and will utilize more detailed measurements of the system state, as described in this paper. Necessary system modifications are expected to be complete in early FY06, with dynamic tests commencing shortly thereafter.

\section{NOMENCLATURE}

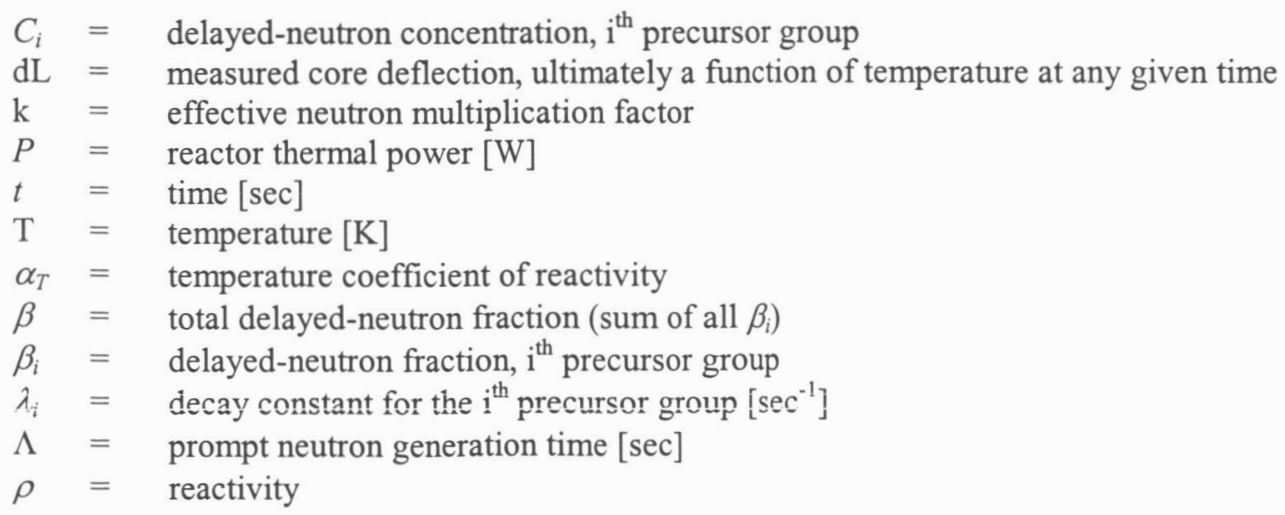




\section{ACKNOWLEDGMENTS}

NASA's Project Prometheus supported the work described within this paper, in whole or part, as part of the program's technology development and evaluation activities. Any opinions expressed are those of the author and do not necessarily reflect the views of Project Prometheus.

The author is a Technical Staff Member at Los Alamos National Laboratory currently on assignment at the NASA Marshall Space Flight Center, Huntsville, AL.

\section{REFERENCES}

Bragg-Sitton, S.M., "Heat Pipe Reactor Dynamic Response Tests: SAFE-100a Reactor Core Prototype," in proceedings of the Space Nuclear Conference (SNC-2005), published by the American Nuclear Society, San Diego, CA, 2005, p. $781-789$.

Bragg-Sitton, S.M., and Forsbacka, M., "Application of a Virtual Reactivity Feedback Control Loop in Non-Nuclear Testing of a Fast Spectrum Reactor," in proceedings of the International Congress on Advanced Nuclear Power Plants (ICAPP-2004), American Nuclear Society Order No. 700306, Pittsburgh, PA, 2004, p. 2259-2268.

Godfroy, T.J.; Kapernick, R.; and Bragg-Sitton, S.M., "Thermally Simulated 32kW Direct-Drive Gas-Cooled Reactor: Design, Assembly and Test, " in proceedings of Space Technology and Applications International Forum (STAIF-2004), edited by M. El-Genk, AIP Conference Proceedings 699, New York, 2004, pp. 757-763.

Hetrick, D.L., Dynamics of Nuclear Reactors, The University of Chicago Press, Chicago, IL, 1971.

Stinson-Bagby, K.L. and Fielder, R.S., "Fiber Bragg Gratings for High-Temperature Thermal Characterization," in proceedings of the International Congress on Advanced Nuclear Power Plants (ICAPP-2004), American Nuclear Society Order No. 700306, Pittsburgh, PA, 2004, p. 2288-2294.

Wright, S.A. and Lipinski, R.J., "Pin-Type Gas Cooled Reactor for Nuclear Electric Propulsion," in proceedings of Space Technology and Applications International Forum (STAIF-2003), edited by M. El-Genk, AIP Conference Proceedings 654, New York, 2003, pp. 408-419.

VanDyke, M.K.; Houts, M.G.; Pedersen, K.; Godfroy, T.J.; Dickens, R.; Poston, D.I.; Reid, R.S.; Salvail, P.; and Ring, P., "Phase I Space Fission Propulsion System Testing and Development Progress," in proceedings of Space Technology and Applications International Forum (STAIF-2001), edited by M. El-Genk, AIP Conference Proceedings 552, New York, 2001, pp. 837-842. 